\title{
Incompatibility between E Colicin Plasmids
}

\author{
By PEARL C. COOPER, * FIONA K. L. HAWKINS AND \\ RICHARD JAMES \\ School of Biological Sciences, University of East Anglia, Norwich NR4 7TJ, UK
}

(Received 19 December 1985; revised 7 March 1986)

\begin{abstract}
We have tested the ability of pairs of colicin E plasmids to replicate stably in the same cell line. Although many of the pairs of E colicin plasmids were compatible, plasmids ColE3-CA38, ColE7-K317 and ColE8-J were mutually incompatible, as were ColE5-099, ColE6-CT14 and ColE9-J. Incompatibility between ColE6-CT14 and ColE5-099 or ColE9-J was asymmetrical, whereas incompatibility between the other plasmid pairs was symmetrical.
\end{abstract}

\section{INTRODUCTION}

The $\mathrm{E}$ colicins are a group of plasmid-coded, antibiotic proteins which use the $b t u B$ gene product of Escherichia coli for adsorption to sensitive cells. Nine different colicins belong to this group, distinguished on the basis of immunity tests (Males \& Stocker, 1982; Cooper \& James, 1984). The plasmids which determine these colicins are small, multicopy and non-conjugative (Mock \& Pugsley, 1982; Cooper \& James, 1984). Restriction mapping has revealed similarities between the plasmids which determine eight of these colicins, E2 to E9 (Lawrence \& James, 1984; Watson et al., 1985; Chak \& James, 1986), whereas the colicin E1 plasmid, ColE1-K30, is structurally distinct (see Chan et al., 1985). Colicins E2 to E9 are antigenically related, unlike colicin E1 (Mock \& Pugsley, 1982; Cooper \& James, 1984). We are particularly interested in the relationship of the colicin plasmid ColE8-J with the ColE3-CA38 and with the ColE6-CT14 plasmids, as the latter two plasmids also carry a colicin E8 immunity gene (Chak \& James, 1984; Lawrence, 1984), and in the relationship between the ColE5-099 and the ColE9-J plasmids, both of which carry a colicin E5 immunity gene (Chak \& James, 1986).

Plasmid incompatibility, as observed by the unstable inheritance of two plasmids in the same cell line, indicates a common regulatory mechanism for plasmid replication and inheritance and is widely used as a criterion for relatedness between plasmids, such as R plasmids (Datta, 1975). Incompatibility relationships between ColE plasmids have not been examined except for a report of low level incompatibility between ColE2 and ColE3 plasmids (Inselburg, 1974). We have performed plasmid incompatibility tests between pairs of a series of $\mathrm{E}$ colicin plasmids.

\section{METHODS}

Bacterial strains and plasmids. The strains of E. coli used in this work are listed in Table 1 . We are grateful to $\mathbf{K}$. Chak, E. Lederberg (Plasmid Reference Center, Stanford University, Calif., USA) and M. Lawrence for supplying us with plasmid-containing strains.

Mating conditions. The R plasmid R64-11, which confers tetracycline resistance $\left(\mathrm{Tc}^{\mathrm{R}}\right)$, was used to mobilize ColE plasmids into recipient cells already harbouring a ColE plasmid. Colicin E-resistant (btuB) strains were used to avoid colicin-killing of non-immune cells. The donor and recipient strains were grown separately to exponential phase to a cell density of approximately $2 \times 10^{8} \mathrm{cells} \mathrm{ml}^{-1}$, mixed in a ratio of $1: 1$, incubated without shaking for $2 \mathrm{~h}$ and then plated on medium containing tetracycline $\left(10 \mu \mathrm{g} \mathrm{m}^{-1}\right)$ and a counterselective agent, either nalidixic acid $\left(25 \mu \mathrm{g} \mathrm{ml}^{-1}\right)$, streptomycin $\left(100 \mu \mathrm{g} \mathrm{ml}^{-1}\right)$ or rifampicin $\left(50 \mu \mathrm{g} \mathrm{ml}^{-1}\right)$. The plates were incubated for 1-2 d. All incubations were at $37^{\circ} \mathrm{C}$.

Testing for incompatibility. With native plasmids this was tested at three stages, with no selection for the maintenance of either plasmid. Approximately $20 \mathrm{Tc}^{R}$ transconjugant colonies obtained from each mating were 
Table 1. E. coli strains

\begin{tabular}{lll} 
Strain & \multicolumn{1}{c}{ Relevant properties } \\
\multicolumn{4}{c}{ Recipients in conjugation } \\
PC702 & W3110 btuB gyrA (ColE2-P9) \\
PC703 & W3110 btuB rpsL (ColE3-CA38) \\
PC704 & W3110 btuB gyrA (ColE4-CT9) \\
PC705 & W3110 btuB rpsL (ColE5-099) \\
PC706 & W3110 btuB gyrA (ColE6-CT14) \\
PC707 & W3110 btuB rpoB (ColE7-K317) \\
PC708 & W3110 btuB rpsL (ColE8-J) \\
PC709 & W3110 btuB rpsL (ColE9-J) \\
& \multicolumn{1}{c}{ Donors in conjugation } \\
PC710 & W3110 btuB (R64-11) (ColE8-J) \\
PC711 & W3110 btuB (R64-11) (ColE9-J) \\
PC712 & W3110 btuB (R64-11) (ColE2-P9) \\
PC713 & W3110 btuB (R64-11) (ColE3-CA38) \\
PC714 & W3110 btuB (R64-11) (ColE5-099) \\
PC715 & W3110 btuB (R64-11) (ColE6-CT14) \\
PC716 & W3110 btuB (R64-11) (ColE7-K317) \\
& \multicolumn{3}{c}{ Colicin indicator bacteria } \\
PRC594 & W3110 (ColE2-P9); colicin E2 immune \\
KC10 & W3110 (pKC10); colicin E3 immune & \\
PRC625 & W3110 (ColE4-CT9); colicin E4 immune & E. Lederberg \\
PRC596 & W3110 (ColE5-099); colicin E5 immune & E. Lederberg \\
ML8412 & W3110 (pML8412); colicin E6 immune & E. Lederberg \\
PRC597 & W3110 (ColE7-K317); colicin E7 immune & Lawrence (1984) \\
PC651 & W3110 (ColE8-J); colicin E8 immune \\
KC65 & W3110 (pKC65); colicin E9 immune & Chak \& James (1985) \\
& * Where no source is shown, the strain was from this laboratory. \\
&
\end{tabular}

tested for the production of the colicins determined by each ColE plasmid, using indicator strains which were specifically immune to one or the other colicin (stage 1). Between four and eight transconjugants producing both colicins were purified by streaking on Tc plates and two to four individual colonies from each streak were tested for colicin production (stage 2). Two single colonies producing both colicins were inoculated into separate broths $(5 \mathrm{ml})$, which were grown overnight to stationary phase and then plated for single colonies. Between 15 and 20 single colonies were then tested for colicin production (stage 3). Plasmid DNA was isolated from certain stage 3 broth cultures by the method of Birnboim \& Doly (1979), and was electrophoresed on $0.7 \%$ Tris/acetate agarose gels. Not all pairs of plasmids can be easily distinguished on the basis of mobility in agarose gels because of the small differences in their $\boldsymbol{M}_{\mathrm{r}}$. Incompatibility tests between all plasmids pairs were done at least twice, with reproducible results.

Media, colicin testing and electrophoresis. The media used, the method for testing colicin production and the electrophoresis conditions for plasmid DNA have been previously described (Cooper \& James, 1984).

\section{RESULTS AND DISCUSSION}

\section{Incompatibility tests between ColE plasmids}

We used as the criterion for compatibility the ability of transconjugant colonies and their progeny to produce colicins determined by each of the pair of ColE plasmids under test. Colicin E-resistant $(b t u B)$ strains were used so that there was no selection pressure in favour of maintenance of colicin plasmids for immunity. $\operatorname{rec} A$ strains, often used in incompatibility studies to avoid the possibility of recombination between plasmid pairs, were unsuitable for use with ColE plasmids because colicin production is reduced in the absence of the recA gene product (Helinski \& Herschman, 1967). Mock \& Pugsley (1982) found that the recombination frequency between the pairs of ColE plasmids which they constructed by R64-11 mobilization is of the order of $10^{-4}$ per transconjugant. We considered this negligible in the present study. 
Table 2. Incompatibility reactions between native ColE plasmids

c, Compatibility; I, incompatibility

Plasmid donated

ColE plasmid present in recipient:

with R64-11

\begin{tabular}{|c|c|c|c|c|c|c|c|}
\hline \multirow[t]{5}{*}{ E2 } & E3 & E4 & E5 & E6 & E7 & E8 & E9 \\
\hline & C & & & & & C & \\
\hline & & & & & I & I & \\
\hline & & & & I & & C & I \\
\hline & C & & I & & C & $\mathbf{C}$ & I \\
\hline C & I & C & & $\mathrm{C}$ & & I & C \\
\hline C & I & C & C & $\mathrm{C}$ & I & & C \\
\hline $\mathrm{C}$ & C & C & I & I & C & $\mathrm{C}$ & \\
\hline
\end{tabular}

Furthermore, we saw no evidence of recombinant formation on agarose gels of plasmids extracted from strains producing two colicins (data not shown).

(a) Incompatibility tests with ColE8-J. R64-11 mobilized ColE8-J into recipients harbouring plasmids encoding colicins E2, E3, E4, E5, E6, E7 and E9. Between $40 \%$ and $60 \%$ of recipients acquiring $\mathrm{Tc}^{\mathrm{R}}$ also became ColE8 ${ }^{+}$. Transconjugant colonies which were $\mathrm{ColE} 8^{+}$and either $\mathrm{ColE}^{+}, \mathrm{ColE}^{+}, \mathrm{ColE}^{+}, \mathrm{ColE}^{+}$or ColE9 ${ }^{+}$gave stable cell lines in which $>90 \%$ of colonies tested produced both colicins at stage 2 and at stage 3, indicating compatibility between the respective ColE plasmids. We already knew that ColE8-J and ColE9-J were compatible, because they were both present in the same natural isolate of $E$. coli (Cooper \& James, 1984).

When transconjugant colonies which were ColE8 ${ }^{+}$and $\mathrm{ColE}^{+}$, or $\mathrm{ColE} 8^{+}$and $\mathrm{ColE} 7^{+}$, were subcultured, fewer than $10 \%$ of the colonies tested at stage 2 or stage 3 produced both colicins. The majority of segregants produced the colicin encoded by the plasmid originally resident in the recipient cells. In reciprocal crosses, R64-11 mobilized ColE3-CA38 and ColE7-K317 into ColE $8^{+}$recipients, but on subculture fewer than $10 \%$ of colonies tested produced two E colicins. The majority of segregants in these two crosses produced colicin E8, indicating that incompatibility between these plasmids is symmetrical. Reciprocal crosses between ColE $3^{+}$and ColE7 ${ }^{+}$strains gave similar results, indicating that ColE3-CA38, ColE7-K317 and ColE8-J belong to the same plasmid incompatibility group (Table 2). Mock \& Pugsley (1982) constructed cell lines carrying two ColE plasmids for recombination studies. They found that ColE3-CA38 could apparently stably co-exist with ColE2-P9, ColE4-CT9, ColE5-099 and ColE6-CT14, but not with ColE7-K317. Inselburg (1974) reported that the frequency of segregation of Col plasmids in a strain carrying both ColE2 and ColE3 was approximately $1 \%$. These plasmids would not be classified as incompatible by our criteria.

ColE3-CA38 and ColE8-J bear striking structural similarities, as determined by restriction mapping and subcloning (Chak \& James, 1984; Lawrence \& James, 1984). Colicin E3-CA38 has RNAase activity (Bowman et al., 1971; Senior \& Holland, 1971), whereas indirect evidence has shown that colicin E8-J has DNAase activity (Lawrence, 1984), like colicin E2-P9 (Schaller \& Nomura, 1976). Colicin E7-K 317 is also believed to have DNAase activity (Mock \& Pugsley, 1982). Restriction maps of ColE7-K317 show fewer similarities to ColE3-CA38 than to ColE2P9 (Watson et al., 1985). We found ColE2-P9 to be compatible with ColE7-K317 (Table 2).

(b) Incompatibility tests with ColE9-J. R64-11 mobilized ColE9-J into all ColE ${ }^{+}$recipients tested, except ColE6-CT14 (Table 2). Between $40 \%$ and $60 \%$ of $\mathrm{Tc}^{\mathrm{R}}$ transconjugant colonies produced colicin E9 in addition to the colicin specified by the resident plasmid. ColE9-J was compatible with all but ColE5-099, with $>90 \%$ of colonies tested producing both E colicins at stage 2 and stage 3. With ColE5-099 fewer than $10 \%$ of colonies tested were ColE5 ${ }^{+}$and ColE9 ${ }^{+}$ at stage 2 and stage 3 , indicating plasmid incompatibility. In the reciprocal cross, in which the ColE5-099 plasmid was mobilized into a ColE9-J recipient, the plasmid pair segregated during subculture. In both crosses the majority of segregants produced the colicin determined by the resident plasmid, indicating symmetrical incompatibility. 
Although ColE9 ${ }^{+}$transconjugants were not obtained with a $\mathrm{ColE6}^{+}$recipient, in the reciprocal cross, ColE6-CT14 was mobilized into a ColE9+ recipient. However, none of the transconjugants which were $\mathrm{ColE}^{+}$at stage 1 (approximately $60 \%$ of the $\mathrm{Tc}^{\mathrm{R}}$ transconjugants tested) also produced colicin E9, suggesting that ColE6-CT14 displaced the resident ColE9-J. A similar pattern of asymmetrical incompatibility was observed with ColE5-ColE6 matings. ColE5 either did not enter ColE6 ${ }^{+}$recipients, or did not become established, whereas ColE6 displaced ColE5 in the reciprocal cross.

The structural and functional maps of ColE5-099 and ColE9-J are very similar (Chak \& James, 1986) and the incompatibility results further suggest relatedness. We were surprised by the incompatibility of ColE6 with ColE5 and ColE9. Although ColE6-CT14 shares some restriction sites with these two plasmids, it is some $4 \mathrm{~kb}$ larger. Further, ColE6-CT14 is known to have certain properties in common with ColE3-CA38, e.g. a unique KpnI restriction site located within the respective immunity genes (Lawrence, 1984; Watson et al., 1985) and the presence of a colicin E8 immunity gene (Chak \& James, 1984; Lawrence, 1984). Both colicins also inhibit protein synthesis (Mock \& Pugsley, 1982). ColE3-CA38 and ColE6-CT14, however, are compatible (Table 2).

In the case of ColE1, incompatibility has been shown to be the consequence of a copy number control mechanism which is mediated by the interaction of a small, plasmid-specific, nontranslated RNA species, RNA1, with the RNA primer, RNAII, at the origin of replication (Tomizawa \& Itoh, 1981). We have cloned fragments of ColE8-J and ColE9-J which determine incompatibility (unpublished data). These will be invaluable in helping to understand the molecular basis of the incompatibility relationships described in this paper.

We wish to thank Kin Chak, Mark Lawrence and Derek Lydiate for helpful discussions during the course of this work.

\section{REFERENCES}

Birnboim, H. C. \& Doly, J. (1979). A rapid alkaline extraction procedure for screening plasmid DNA. Nucleic Acids Research 7, 1513-1523.

Bowman, C. M., Dahlberg, J. E., Ikemura, T., Konisky, J. \& NomUra, M. (1971). Specific inactivation of $16 \mathrm{~S}$ ribosomal RNA induced by colicin E3 in vivo. Proceedings of the National Academy of Sciences of the United States of America 68, 964-968.

CHAK, K.-F. \& JAMES, R. (1984). Localization and characterization of a gene on the ColE3-CA38 plasmid that confers immunity to colicin E8. Journal of General Microbiology 130, 701-710.

CHAK, K.-F. \& JAMES R. (1985). Analysis of the promoters for the two immunity genes present in the ColE3-CA38 plasmid using two new promoter probe vectors. Nucleic Acids Research 13, 2519-2531.

CHAK, K.-F. \& JAMES, R. (1986). Characterization of the ColE9-J plasmid and analysis of its genetic organization. Journal of General Microbiology 132, 61-71.

Chan, P. T., OHmori, H., Tomizawa, J. \& Lebowitz, J. (1985). Nucleotide sequence and gene organization of ColEl DNA. Journal of Biological Chemistry 260, 8925-8935.

CoOPer, P. C. \& James, R. (1984). Two new E colicins, E8 and E9, produced by a strain of Escherichia coli. Journal of General Microbiology 130, 209-215.

DATTA, N. (1975). Epidemiology and classification of plasmids. In Microbiology 1974, pp. 9-15. Edited by D. Schlessinger. Washington, DC: American Society for Microbiology.

Helinski, D. R. \& Herschman, H. R. (1967). Effect of $\mathrm{Rec}^{-}$mutations on the activity of colicinogenic factors. Journal of Bacteriology 94, 700-706.
INSELBURG, J. (1974). Incompatibility exhibited by colicin plasmids E1, E2 and E3 in Escherichia coli. Journal of Bacteriology 119, 478-483.

LAWRENCE, G. M. P. (1984). Characterisation of the ColE8-J plasmid. PhD thesis, University of East Anglia, Norwich, UK.

LAWRenCE, G. M. P. \& JAMEs, R. (1984). Characterisation of the ColE8 plasmid, a new member of the group E colicin plasmids. Gene 29, 145-155.

Males, B. M. \& Stocker, B. A. D. (1982). Colicins E4, E5, E6 and A and properties of btuB $B^{+}$colicinogenic transconjugants. Journal of General Microbiology 128 , 95-106.

Mock, M. \& Pugsley, A. P. (1982). The btuB group $\mathrm{Col}$ plasmids and homology between the colicins they encode. Journal of Bacteriology 150, 1069-1076.

Schaller, K. \& Nomura, M. (1976). Colicin E2 is a DNA endonuclease. Proceedings of the National Academy of Sciences of the United States of America 73, 3989-3993.

Senior, B. W. \& Holland, I. B. (1971). Effect of colicin E3 upon the $30 \mathrm{~S}$ ribosomal subunit of Escherichia coli. Proceedings of the National Academy of Sciences of the United States of America 68, 959963.

Tomizawa, J. \& IтоH, T. (1981). Plasmid ColE1 incompatibility determined by interaction of RNA 1 with primer transcript. Proceedings of the National Academy of Sciences of the United States of America 78, 6096-6100.

WATSON, R. J., VERnet, T. \& Visentin, L. P. (1985). Relationships of the Col plasmids E2, E3, E4, E5, E6 and E7: restriction mapping and colicin gene fusions. Plasmid 13, 205-210. 